\title{
Rectal absorption of short chain fatty acids in the absence of chloride
}

\author{
N. I. MCNEIL ${ }^{1}$, J. H. CUMMINGS, AND W. P. T. JAMES \\ From the MRC Dunn Clinical Nutrition Centre, Addenbrooke's Hospital, Cambridge
}

SUMMARY The absorption of short chain fatty acids by the human rectum in the absence of chloride has been studied using a dialysis bag technique. Absorption rates of sodium, water, and short chain fatty acids were unchanged on replacing chloride with sulphate. Final concentrations of bicarbonate were $35.4 \pm 3.4 \mathrm{mmol} / \mathrm{l}$ in the presence of chloride and $37 \cdot 1 \pm 5.2 \mathrm{mmol} / \mathrm{l}$ in the absence of chloride. The rates of movement of bicarbonate into the dialysates were similar. If bicarbonate is secreted in exchange for chloride then it also appears to be secreted in exchange for absorbed short chain fatty acids.

When saline solutions are perfused through the human colon more chloride than sodium is absorbed and ionic balance is maintained by the secretion of bicarbonate (Devroede and Phillips, 1969; Bown et al., 1972; Rask-Madsen, 1973). In animal studies these features of sodium and chloride absorption were first described for rat colon by Parsons in 1956. Devroede and Phillips (1969) and Bown and his colleagues (1972) have suggested that there is a chloride-bicarbonate exchange in the human colon with 'bicarbonate secretion obligatorily coupled to chloride absorption'.

Although chloride is a major ionic constituent of ileal contents (Phillips and Giller, 1973), it is present in only low concentrations in human faeces (Wrong et al., 1965; Bjork et al., 1976; Cummings et al., 1976) where the major anion is short chain fatty acid (Rubinstein et al., 1969; Bjork et al., 1976). In an initial series of studies of short chain fatty acid absorption by the human large intestine we found that the net absorption rate of short chain fatty acids exceeded that of sodium. This excess was approximately matched by the appearance of bicarbonate (McNeil et al., 1978), which implies that short chain fatty acids may be an alternative anion available for exchange with bicarbonate in the large intestine.

To observe the effect of chloride on these exchanges we conducted studies on short chain fatty acid and bicarbonate movement in the presence of

\footnotetext{
${ }^{1}$ Present address and address for correspondence: Department of Medicine, University College Hospital Medical School, The Rayne Institute, University Street, London WC1E 6JJ.

Received for publication 2 November 1978
}

sulphate rather than chloride. If the absorption of short chain fatty acid is dependent on chloridebicarbonate exchange one would expect a fall in absorption in the absence of chloride.

The use of sulphate, which is unabsorbed by the human colon, also allowed an assessment of the validity of the dialysis bag technique, as a large loss of the sulphate would indicate exchange of dialysis fluid with a pool of fluid in the lumen rather than across the mucosa.

\section{Methods}

Seventeen subjects (16 women, one man: aged 24-66 years) who were subjects attending an obesity clinic volunteered to take part in the study. None had colonic disease or gastrointestinal symptoms at the time of the study, for which they were not fasted. Subjects were studied for two separate but consecutive hours, the solutions being alternated in order of use. After discarding faecally stained and burst bags, there were seven chloride containing studies and six sulphate studies for analysis, four of each being paired.

Absorption was measured using a length of $8 / 32$ dialysis tubing mounted on a stiffened catheter, which after being filled was introduced into the rectum and left for one hour (Edmonds, 1971). The average dimensions of the filled dialysis bags were $8 \times 0.5 \mathrm{~cm}$ and average volume $2.5 \mathrm{ml}$.

The solutions used for this study are shown in Table 1. Potassium chloride in solution 1 was completely replaced by potassium sulphate in solution 2 and the $\mathrm{pH}$ of each solution adjusted with hydro- 
Table 1 Change in composition of trial solutions after one hour's rectal dialysis (mean $\pm S E$ )

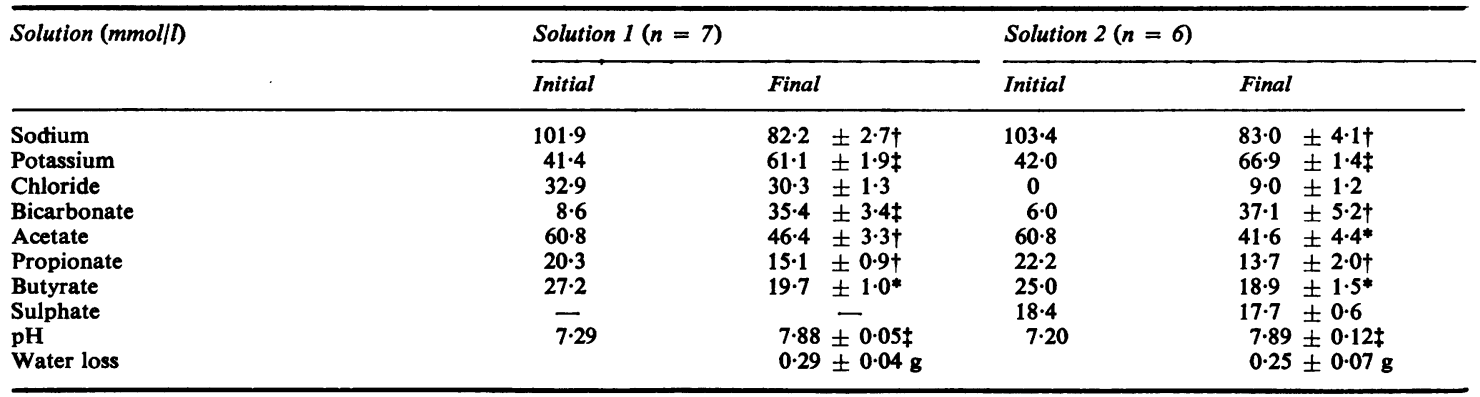

*Differ from initial values, ${ }^{*} P<0.02, \dagger P<0.01$, $¥ \mathrm{P}<0.001$.

chloric or sulphuric acid respectively.

Short chain fatty acids, sodium, potassium, chloride, bicarbonate, $\mathrm{pH}$, and water loss were measured as previously described (McNeil et al., 1978).

Sulphate was measured by microtitration against lead perchlorate, free lead ion being detected by a specific lead ion electrode (Orion Research Ltd). Initially the samples were acidified with perchloric acid and incubated overnight in Conway units (Conway, 1957). The outer well contained 2M potassium hydroxide solution for absorbing all the short chain fatty acids present in the sample. Without this separation technique we found that the short chain fatty acids interfered with the sulphate analysis. The centre well was emptied into a beaker and the solution made up to $20 \mathrm{ml}$, of which $50 \%$ was analytical grade methanol. The $\mathrm{pH}$ was adjusted to $\mathrm{pH} 7 \cdot 2-7 \cdot 3$ and the lead specific electrode allowed to equilibrate in this lead-free solution until a constant potential was attained. A constant rate infusion of lead perchlorate solution was started. The sulphate concentration was determined by comparing the time to reach the end-point of the titration with end-point times of a series of standard sulphate solutions.

The amount of sulphate lost from the bags was determined from initial and final weights in the bags and the initial and final sulphate concentration. The volume of any potential fluid space between the bag and the mucosa was calculated by dividing the amount of sulphate apparently lost from the dialysis bag by the final dialysate sulphate concentration.

Statistical analyses are by Student's $t$ test.

\section{Results}

The initial and final concentrations of the constituents of the solutions used are shown in Table 1 For solution 1, the solution containing chloride, there was a fall in the concentration of sodium, chloride, and acetic, propionic, and butyric acids.
Table 2 Rates of movement of ions through dialysis tubing during one hour's rectal dialysis ( $\mu \mathrm{mol} / \mathrm{sq} \mathrm{cm} / \mathrm{h}$ )

\begin{tabular}{lrr}
\hline & Solution 1 & \multicolumn{1}{c}{ Solution 2} \\
\hline Sodium & $6.5 \pm 0.9$ & $6.2 \pm 0.6$ \\
Potassium & $-2.8 \pm 0.3$ & $-3.6 \pm 0.4$ \\
Chloride & $1.4 \pm 0.3$ & $-1.7 \pm 0.2$ \\
Bicarbonate & $-5.0 \pm 0.7$ & $-5.5 \pm 0.9$ \\
Acetate & $4.4 \pm 0.6$ & $4.9 \pm 0.9$ \\
Propionate & $1.5 \pm 0.2$ & $2.1 \pm 0.4$ \\
Butyrate & $2.2 \pm 0.4$ & $1.7 \pm 0.4$ \\
Sulphate & & $0.6 \pm 0.2$ \\
Water $(\mathrm{mg} / \mathrm{sq} \mathrm{cm} / \mathrm{h})$ & $25.8 \pm 4.1$ & $21.9 \pm 5.5$ \\
\hline
\end{tabular}

一: secretion.

The concentrations of potassium and bicarbonate rose. Mean water loss during one hour's dialysis was $0.29 \mathrm{~g}$ and $\mathrm{pH}$ rose from $\mathrm{pH} 7.29$ to 7.88 during this period in the rectum.

The rates of movement through the dialysis membrane of the ions and water have been calculated from these concentrations (Table 2) and show that sodium, chloride, water, and the three short chain fatty acids are absorbed. Simultaneously potassium and bicarbonate pass into the dialysate. These findings are similar to our previously reported findings for a similar solution (McNeil et al., 1978).

When the chloride free solution was used (Table 1) the final concentrations of sodium, acetate, propionate, and butyrate were insignificantly different from solution 1. Similar increases in the concentration of potassium and bicarbonate occurred. The final chloride concentration was $9.0 \mathrm{mmol} / \mathrm{l}$ and sulphate concentration fell from 18.4 to $17.7 \mathrm{mmol} / \mathrm{l}$. Water loss and pH changes were similar to solution 1.

Net absorption of sodium, water, acetate, propionate, and butyrate (Table 2) was similar for the two solutions. These ionic movements occurred despite a reversal in chloride movement. In particular, final bicarbonate concentrations did not differ in the two solutions. The mean loss of sulphate during one hour's dialysis was $12 \cdot 8 \pm 5.4 \%$ of the amount of sulphate initially in the bag. This exceeded the 
mean water loss of $10.0 \pm 1.8 \%$. The potential fluid space in contact with the bag at the end of the dialysis period with the sulphate containing bags was calculated to be $0.4 \pm 0.1 \mathrm{ml}$.

\section{Discussion}

These studies conducted in the normal rectum show that the complete replacement of chloride by sulphate did not reduce the rates of absorption of acetic, propionic, and butyric acids. The final concentration of bicarbonate was the same in the two studies and rates of movement of bicarbonate from the mucosa to the dialysate were unchanged.

In studies of colonic absorption by the perfusion technique when equimolar sodium chloride solutions are used, Devroede and Phillips (1969) found a significantly greater absorption of chloride than sodium and that 'the difference was closely approximated by secretion of bicarbonate'. Rask-Madsen (1973) described similar findings after his colonic perfusion studies. Bown et al. (1972) in studies on the excluded human colon considered 'that bicarbonate secretion is obligatorily coupled to chloride absorption'. When chloride was replaced in Bown's studies by sulphate, bicarbonate secretion was replaced by bicarbonate absorption. In this paper the replacement of chloride by sulphate had no such effect on bicarbonate movement. This implies that, if there is a coupled transport mechanism for anions, it is not specific for chloride and that short chain fatty acids are an alternative anion for exchange with bicarbonate. The presence of short chain fatty acids in our test solution is the major difference between this study and the study of Bown et al. (1972) and probably accounts for the differences found. Bown et al. (1972) did perfuse the whole colon, whereas we have studied the rectum only. This is another major difference between the studies. However, we do not believe that regional differences in colonic function account for the discrepant results, as in other studies that we have performed (unpublished) short chain fatty acids are absorbed throughout the length of the colon.

Our findings are in agreement with those of Schmitt et al. (1977). In a study of short chain fatty acid absorption in the human ileum, they describe a rise in $\mathrm{pH}$ of the perfusate and consider that bicarbonate is secreted in exchange for some of the absorbed short chain fatty acids.

An alternative source of bicarbonate has been suggested by Stevens and his colleagues (Argenzio et al., 1977). After a study of short chain fatty acid absorption in the equine large intestine they proposed that short chain fatty acids may be absorbed in the unionised form. The supply of $\mathrm{H}^{+}$for this may in part come from a sodium-hydrogen exchange by the mucosa and in part from the formation of bicarbonate and $\mathrm{H}^{+}$from the large quantities of carbon dioxide present in the lumen. By this means any absorption of short chain faity acid in excess of sodium absorption will be matched by the appearance of an equal amount of bicarbonate in the lumen. Either of these explanations for the appearance of bicarbonate is compatible with our findings.

The use of sulphate which is unabsorbed in the colon indicates from the loss of sulphate from the dialysis bag that there is a small fluid space in the rectum. This space measures $0.4 \pm 0.1 \mathrm{ml}$ and is possibly in crypts, mucus, and over the mucosal surface. The presence of the catheter in the rectum may promote the secretion of mucus. Recalculation of net rates of absorption or secretion using sulphate as a non-absorbable marker reduces the net flux rates of those ions that were absorbed (sodium and the short chain fatty acids), and increases the rates of movement of potassium and bicarbonate into the dialysis bags. However the final concentrations in the chloride and sulphate containing solutions are not changed significantly by this calculation, so similar conditions must exist during the rectal dialysis of either solution.

In conclusion, the replacement of chloride by sulphate in the lumen does not reduce short chain fatty acid or sodium absorption in the human rectum and chloride is not essential to bicarbonate handling by the human large intestine.

We are grateful to $\mathrm{Dr} H$. S. Wiggins for his considerable assistance in the measurement of sulphate.

\section{References}

Argenzio, R. A., Southworth, M., Lowe, J. E., and Stevens, C. E. (1977). Interrelationship of $\mathrm{Na}, \mathrm{HCO}_{3}$, and volatile fatty acid transport by equine large intestine. American Journal of Physiology, 233, E469-E478.

Bjork, J. T., Soergel, K. H., and Wood, C. M. (1976). The composition of 'free' stool water. (Abstract.) Gastroentero$\log y, 70,864$.

Bown, R. L., Sladen, G. E., Rousseau, B., Gibson, J. A., Clarke, M. L., and Dawson, A. M. (1972). A study of water and electrolyte transport by the excluded human colon. Clinical Science, 43, 891-902.

Conway, E. J. (1957). Microdiffusion Analysis and Volumetric Error. 4th ed. Crosby Lockwood: London.

Cummings, J. H., Hill, M. J., Jenkins, D. J. A., Pearson, J. R., and Wiggins, H. S. (1976). Changes in fecal composition and colonic function due to cereal fiber. American Journal of Clinical Nutrition, 29, 1468-1473.

Devroede, G. J., and Phillips, S. F. (1969). Conservation of sodium, chloride, and water by the human colon. Gastroenterology, 56, 101-109.

Edmonds, C. J. (1971). Absorption of sodium and water by human rectum measured by a dialysis method. Gut, 12 , 356-362. 
McNeil, N. I., Cummings, J. H., and James, W. P. T. (1978). Short chain fatty acid absorption by the human large intestine. Gut, 19, 819-822.

Parsons, D. S. (1956). The absorption of bicarbonate-saline solutions by the small intestine and colon of the white rat. Quarterly Journal of Experimental Physiology, 41, 410-420.

Phillips, S. F., and Giller, J. (1973). The contribution of the colon to electrolyte and water conservation in man. Journal of Laboratory and Clinical Medicine, 81, 733-746.

Rask-Madsen, J. (1973). Simultaneous measurement of electrical polarization and electrolyte transport by the entire normal and inflamed human colon during in vivo perfusion. Scandinavian Journal of Gastroenterology, 8,
327-336.

Rubinstein, R., Howard, A. V., and Wrong, O. M. (1969). In vivo dialysis of faeces as a method of stool analysis; IV: the organic anion component. Clinical Science, 37, 549-564.

Schmitt, M. G., Jr., Soergel, K. H., Wood, C. M., and Steff, J. J. (1977). Absorption of short-chain fatty acids from the human ileum. American Journal of Digestive Diseases, 22, 340-347.

Wrong, O. M., Metcalfe-Gibson, A., Morrison, R. B. I., $\mathrm{Ng}$, S. T., and Howard, A. V. (1965). In vivo dialysis of faeces as a method of stool analysis. I. Technique and results in normal subjects. Clinical Science, 28, 357-375. 